\title{
Propuesta metodológica para la identificación del valor agregado como input de Lean Services en instituciones de educación superior
}

\author{
Joseph Robert Voelkl Peñaloza*, Jorge Alberto Silva Rueda*, \\ Clara Mabel Solano Vanegas*, Giovanna Rosa Fiorillo Obando* \\ Pontificia Universidad Javeriana. Bogotá, Colombia
}

Recibido: 29 de abril de 2014 / Aprobado: 30 de junio de 2014

Resumen: Las empresas utilizan Lean Thinking como filosofía para eliminar desperdicios. Asimismo, CRM es una estrategia de negocio para mejorar la relación con los clientes, aunque su potencial no se ha desarrollado completamente. Desde esta visión, se busca administrar las expectativas de los clientes, incluyendo el valor agregado (VA). De allí surge la pregunta: ¿de qué manera la compañía define el VA esperado por los clientes? Como respuesta se plantea una propuesta metodológica para identificar el VA esperado como input de Lean, en instituciones de educación superior.

Palabras clave: valor agregado / CRM / técnica de pensamiento esbelto / instituciones de educación superior / eficiencia organizacional / satisfacción del cliente

\section{Methodological proposal to identification of value added as input Lean Services, in higher education institutions}

Aвstract: Companies use Lean Thinking philosophy to eliminate wastes. Furthermore, CRM is a business strategy to improve the relationship with customers; although its potential has not been developed completely. From this perspective seeks to manage the customer's expectations including what they expect as VA. At this point, the question is How does a company define the customers expected VA? As an alternative answer this research presents a methodological proposal to identify the expected VA as Lean input, applied at academic institutions.

Key words: value added / CRM / Lean Thinking technique / higher education institutions / organizational efficiency / customer satisfaction

* Correos electrónicos: jrvoelkl@javeriana.edu.co, solano.c@javeriana.edu.co, fiorillo@javeriana.edu.co, jsilva@javeriana.edu.co 


\section{INTRODUCCIÓN}

Womack y Jones (2003) describieron el Lean Thinking (o pensamiento esbelto) como el antídoto a los muda ('desperdicios' en japonés), debido a que provee una manera de especificar el valor, alineando las acciones que lo crean en la mejor secuencia posible, ejecutando estas sin interrupción cuando alguien las requiera y llevándolas a cabo de la manera más efectiva. Este es el propósito de la filosofía del Lean Thinking, el cual debería ser la meta de cualquier tipo de empresa que quisiera aplicarla, sin importar su naturaleza (manufacturera o de servicios). En la actualidad, Lean, como filosofía, tiene ventajas no solo en la reducción o eliminación de los desperdicios; algunas de estas son el aumento en la participación y colaboración de los empleados, mejores productos, satisfacción del cliente, etcétera.

A su vez, Lean Services, como parte de Lean Thinking, busca eliminar y reducir los desperdicios no solo en el flujo del material sino en lo que respecta al flujo de información y de documentos alrededor de este, incluidas las formas tangibles e intangibles propias del negocio, con la tecnología de información adecuada para tal efecto.

Precisamente, la razón de ser del negocio de cualquier empresa es satisfacer las necesidades y expectativas de sus clientes, las cuales son muy variables, en particular para las empresas de servicios, porque cada cliente tiene diversos requerimientos y diferentes percepciones del servicio suministrado. Por esta razón, las empresas se cuestionan sobre qué es lo que el cliente quiere.

Para responder, se requiere preguntarle directamente al cliente; sin embargo, esto solo es posible si la empresa tiene relación y/o comunicación con este, que no esté basada únicamente en el dato de contacto, sino que tenga en cuenta su percepción y lo que él espera de la compañía. De esta forma, a la empresa le será más sencillo alinear sus procesos conociendo las expectativas y el enfoque hacia el cliente.

Particularmente, desde la década de 1990, Customer Relationship Management (CRM) empezó a aparecer en el lenguaje de los negocios como el concepto que se debe seguir para fortalecer las relaciones con los clientes, especialmente con los externos. Originalmente, esta estrategia perseguía metas de mercadeo y comerciales; sin embargo, su potencial para cubrir otros campos debe ser desarrollado. 
Como se mencionó, Lean Services necesita identificar la percepción y los requerimientos del cliente - en otras palabras, ¿cuánto es lo que el cliente está dispuesto a pagar? - identificando las actividades que generan valor agregado en el servicio ofrecido y suministrado al cliente. Hoy en día, la identificación de las actividades generadoras de valor se realizan mediante la aplicación de diferentes herramientas, como el mapeo de la cadena de valor, conocido como VSM por sus siglas en inglés (Value Stream Mapping), entre otras. Estas herramientas se basan en la identificación del valor agregado desde la perspectiva interna de la organización, sin tener en cuenta el importante punto de vista del cliente externo. Por esto, los autores consideran importante complementar el proceso de identificación de valor, incluidas la percepción y expectativas de los clientes externos mediante el concepto de CRM.

La barrera encontrada es el hecho de que CRM debe ser diseñado por cada compañía, de acuerdo con sus propias características; no hay una guía puntual y específica, en especial información respecto de los requerimientos y variables que Lean Services pueda utilizar y analizar mediante la aplicación del concepto de CRM; en otras palabras, de qué manera la información que suministra una filosofía de negocio como CRM puede traducirse de modo que les permita a las instituciones tener un diagnóstico desde una óptica externa de los procesos y que sirva como punto de partida para un análisis y rediseño de procesos internos en la institución.

En tal sentido, este artículo es el resultado de una investigación desarrollada para proponer una metodología de diagnóstico del valor agregado, basándose en el concepto de CRM, a manera de insumo para una futura implementación de Lean Services.

\section{DESARROLLO DE LA INVESTIGACIÓN}

\subsection{Aplicaciones documentadas de CRM y su relación potencial con Lean Services}

En la búsqueda de generar valor, Lean Services recurre a diversos instrumentos de diagnóstico, principalmente desde la perspectiva del cliente interno, pese a que - como se especificó- lo que es importante para la organización es la satisfacción del cliente externo; sin embargo, no hay una herramienta desde la filosofía Lean que soporte la determinación y diagnóstico de los requerimientos y expectativas de este; lo anterior soporta el propósito de la investigación. 
CRM es un concepto orientado al cliente y ha sido implementado en la actualidad por muchas instituciones; sin embargo, en el sector académico, su implementación ha sido limitada y en la mayoría de sus aplicaciones se ha traducido en la utilización parcial de su potencial solo para recolección de datos y servicios puntuales, sin una mirada crítica y analítica de la percepción del valor agregado suministrado al cliente externo, identificado como estudiantes potenciales y actuales, y las organizaciones asociadas a los programas de prácticas empresariales y sociales.

La filosofía de Lean Services se orienta al diseño y mejoramiento de los procesos, enfocados a la satisfacción de las expectativas del cliente interno y externo, mediante el servicio ofrecido (Liker \& Morgan, 2006). En este contexto, las empresas han venido contactando directamente a los clientes tratando de conocer sus necesidades y percepciones acerca de sus productos, servicios y usos, aproximación lograda mediante el concepto de CRM.

Mediante la revisión de la información documentada, en relación con las implementaciones de CRM, se encontraron algunas aproximaciones y perspectivas, las cuales de forma muy general y contextualizada al caso particular de la empresa donde se implementó, expresan algunos lineamientos y muestran evidencias de los beneficios que puede llegar a tener este concepto como herramienta de diagnóstico; esta documentación también suministró información de debilidades y fallas que se presentaron en las organizaciones durante los procesos de implementación, las cuales fueron consideradas en la propuesta metodológica como resultado de la investigación.

Por ejemplo, Pan, Tan y Lim (2004) expresan en su artículo acerca del sector público en Singapur, donde se implementó exitosamente CRM en el National IT Literacy Programme (NITLP), que tiene como principal propósito incrementar el nivel de uso de tecnología de la información por la comunidad mediante cursos de capacitación, bajo la consigna gubernamental: Intellingent island for 1992. Para este caso, la organización gubernamental Infocomm Development Authority of Singapore (IDA) redujo la resistencia de la comunidad al uso de tecnología de información, realizando actividades diarias con sus usuarios como resultado del análisis de los datos arrojados por la estrategia de CRM aplicada. Se observaron resultados positivos, como el incremento del número de usuarios y en las respuestas de la investigación de mer- 
cados aplicada posteriormente a su puesta en marcha, en la que las personas entrenadas y capacitadas expresaron sus nuevos intereses en continuar desarrollándose como usuarios con sus nuevas habilidades y a su vez su disposición en participar en grupos de entrenamiento avanzado.

Mendoza, Marius, Pérez y Grimán (2006) establecen que el punto de partida de la implementación de CRM debe ser el estudio de los puntos de interacción o puntos de contacto entre el cliente y la organización. Este análisis debe realizarse inicialmente mediante la caracterización y documentación de los procesos. Además, estos autores mencionan que solo el $30.7 \%$ de las compañías que han implementado modelos CRM han tenido éxito. Los fracasos se han debido principalmente a que no han adoptado de forma integral el concepto y la estrategia de CRM, así se han quedado solo en la implementación de una solución tecnológica sin incluir los tres factores que hacen parte de CRM: el factor humano, los procesos y la tecnología. Siendo aún más explícito, estas implementaciones fallidas ocurrieron por una aproximación errada de CRM, en la que esta es solo vista como una solución de comunicación y no como una estrategia basada en relaciones.

Asimismo, Mendoza et al. (2006) reconocen que para muchas compañías cambiar su orientación estratégica no es tan fácil porque usualmente implica cambiar la orientación de la producción hacia un enfoque al cliente, lo cual obliga a que todos en la organización desarrollen nuevas habilidades. De acuerdo con este nuevo reto de orientar los procesos hacia la satisfacción del cliente, en las instituciones académicas, los estudiantes se convierten en clientes, y como sostienen Seeman y O’Hara (mencionado por Broady-Preston, Felice \& Marshal, 2006), este cambio de "rol" en los estudiantes como clientes permite una ventaja competitiva a la institución desarrollando una habilidad para atraer, retener y servir a sus clientes.

Específicamente en el sector académico, dada la competencia actual en el mercado entre las diferentes universidades, en los ámbitos nacional e internacional, se han realizado algunas incursiones en la implementación de CRM, como lo presentan Broady et al. (2006): casos exitosos documentados como el de la Biblioteca de la Universidad de Malta y la Universidad de Coventry, donde se buscaba una mayor participación de los usuarios, permitiendo a los estudiantes desarrollar 
servicios para las necesidades de la comunidad. En ambos casos, los resultados fueron exitosos, medidos a través del incremento en la percepción y satisfacción de los clientes. Por otra parte, como conclusión particularmente interesante de este estudio, se presenta la sugerencia de involucrar totalmente al personal de las bibliotecas dentro del proceso, estableciendo relaciones personales directas con los usuarios (estudiantes y académicos), enfatizando de esta manera que no solo se trata de poseer información sino de tener en cuenta el factor humano y su interrelación para que cualquier estrategia construida con la información desde CRM sea exitosa.

Otro caso exitoso documentado de implementación de CRM fue la plataforma Right Now Service en la University of South Florida (USF) como herramienta tecnológica para brindar un mejor servicio, a una mayor cantidad de usuarios, permitiéndole al departamento atender más del doble de clientes de lo que usualmente atendía: según el reporte, se pasó de 5,000 estudiantes a 40,000 anualmente, sin incrementar el número de personas contratadas para su atención, según lo describe Beasty (2005).

A su vez, Hilbert, Schonbrunn y Schmode (2007) proponen un concepto de CRM modificado, especialmente orientado a las instituciones que prestan servicios educativos, denominado Student Relationship Management (SRM). Para entender esta aproximación, a continuación se cita la definición que Hilbert et al. (2007, p. 206) realizan acerca del concepto de CRM:

CRM es una orientación estratégica perseguida por todos los miembros de una compañía, con el objeto de incrementar la satisfacción del cliente, su lealtad y su beneficio, como también para la compañía durante toda la relación e interacción entre proveedor-cliente ${ }^{1}$.

1 "CRM is a strategic orientation which is pursued by all members of a company in order to increase customer satisfaction, customer loyalty and benefit for the customer as well as for the company during the entire supplier-customer relationship" (traducción nuestra). 
Como esta definición establece, CRM es una estrategia de negocio cuyos resultados enfocan la organización hacia el cliente, en una relación provechosa a largo plazo. Adicionalmente, como objetivo de CRM está el de lograr una perdurable relación con los clientes actuales que incentive a los nuevos, estrechando sus lazos con la organización. Broady et al. (2006) definen esta relación como "una relación de confianza y cooperación" con los clientes.

Para construir esta relación son necesarios el conocimiento y la información que tiene el cliente; como Lin, Su Hwan-Yann y Chien (2005) mencionan, esta información es muy útil e importante para la organización porque permite el mejoramiento y desarrollo de la cadena de valor influenciada directamente por el cliente, enfocando todos los procesos a este valor agregado esperado y satisfaciendo completamente sus expectativas, incentivando así su lealtad y compromiso con la empresa.

Retomando el concepto de Hilbert et al. (2007), en relación con el modelo SRM, lo que se busca es la satisfacción de los estudiantes y el incremento del valor agregado que se les provee no solo en las actividades académicas sino en todo el proceso, acorde con la filosofía de la institución, lo cual implica un resultado positivo para ambos (el estudiante y la institución), que los lleve a establecer un vínculo a largo plazo, incluso después de su graduación.

También se menciona que para la implementación del CRM es necesaria la definición del ciclo de vida de la relación de la institución con el estudiante, basada en el hecho de que cada fase de este ciclo define las expectativas específicas del cliente.

En este caso, el usuario del servicio, que es el estudiante, para cada una de las fases se propone el Student Relationship Lifecycle (SRLC), como se puede observar en la figura 1. 
Figura 1

Ciclo de vida de la relación con el estudiante

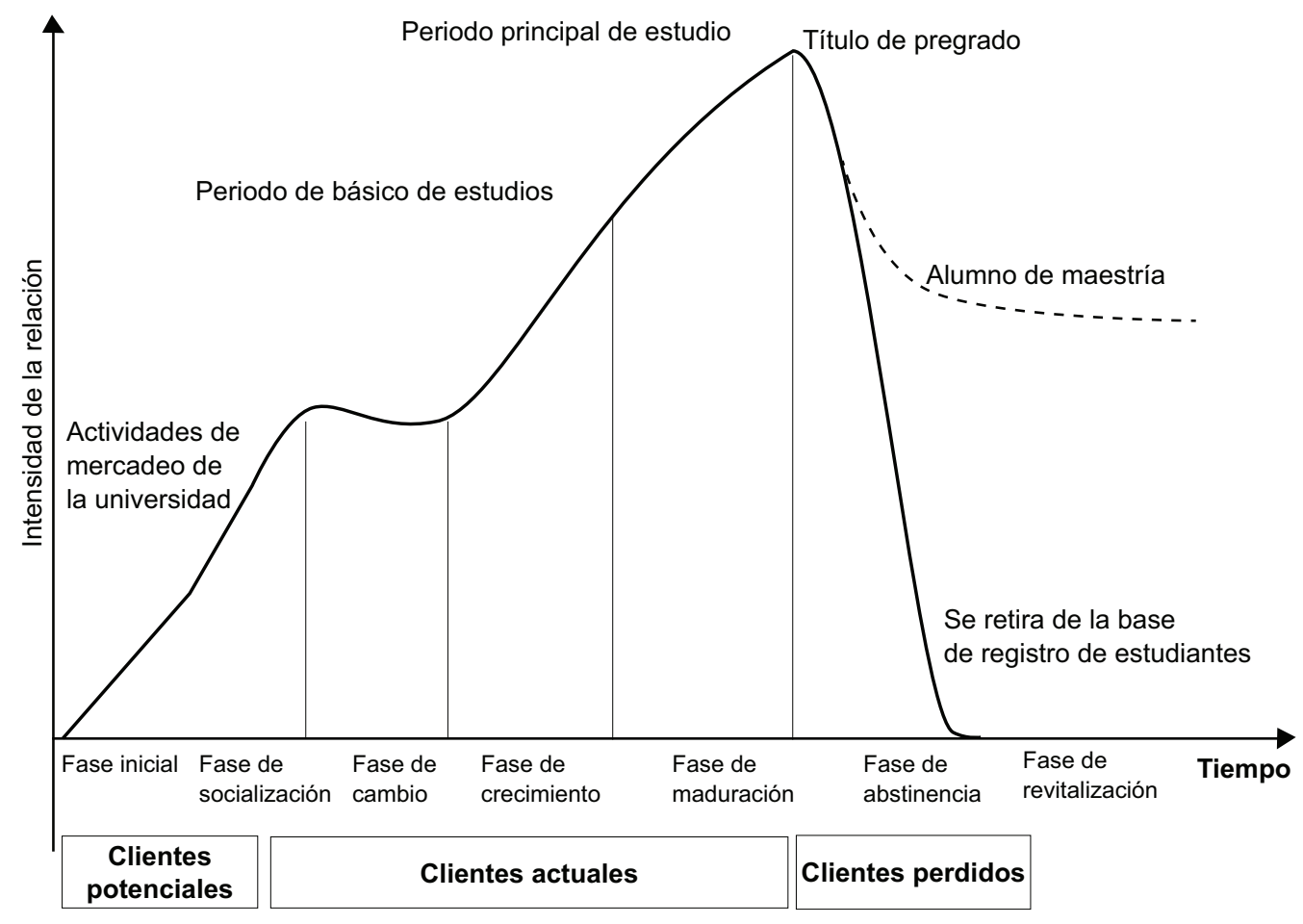

Fuente: Hilbert et al. (2007).

Lo ideal para la estrategia de CRM es que cada institución académica, basada en la información de los estudiantes y el vínculo construido durante sus años de estudios, no permita que decaiga totalmente la relación en la fase de abstinencia y que más bien ingrese en una fase de revitalización, ya sea como estudiantes de posgrados, investigadores y/o profesores de planta o cátedra.

Finalmente, como es posible observar en los estudios revisados previamente, no existen muchas experiencias documentadas de instituciones educativas que hayan implementado el CRM. Un punto interesante y común de las experiencias es que conciben al estudiante como el único cliente de sus servicios, sin considerar a las organizaciones con las que también se interactúa, como en los casos de las prácticas (tanto empresariales como sociales). Por otro lado, no hay experiencias pre- 
vias documentadas acerca del potencial uso del concepto de CRM en la identificación del valor agregado percibido y esperado por los clientes externos, y su aplicación como input ${ }^{2}$ de la implementación de la filosofía Lean Services.

\section{RESULTADOS}

Si bien desde 1990 existe bastante literatura respecto a cómo implementar Lean Manufacturing, en lo referente a servicios recién en la primera década del siglo XXI se ha empezado a concretar las adaptaciones de Lean Manufacturing hacia Lean Service (Abdi, Shavarini \& Hoseini, 2006), o creando nuevas maneras (Roshayati, 2011) de aplicar muchos de los conceptos de sentido común que han de mejorar la eficiencia y productividad de este tipo de procesos. Se destaca en particular que, a pesar de existir varias empresas innovadoras en temas de servicio (Taco Bell, McDonald's, Southwest Airlines), un modelo propuesto para implantar un sistema eficiente aún es incipiente y sirve de base para empezar y en eso se fundamenta la oportunidad de adaptación a un mercado propio (Abdi et al., 2006).

Aunque los esfuerzos apenas han comenzado, ya existen universidades que desde su convicción hacia Lean Manufacturing han incluido en sus currículos el tema de Lean Service como parte inherente de sus programas de Ingeniería Industrial. La Universidad de Alabama (Huntsville, Estados Unidos), además de otras como Cardiff en el Reino Unido, el MIT (Camdridge, Estados Unidos) y Wisconsin-Madison (Estados Unidos), cuenta con programas fuertes y con el respaldo de centros de investigación y consultoría en el tema.

Esto, sin embargo, aún no se traduce en modelos comprobados o adaptaciones probadas de las herramientas Lean en el sector servicios académicos. De hecho, la literatura menciona esfuerzos de aplicación aún dispersos de Lean, sea en el desarrollo de actividades académicas como la aprobación de nuevos contenidos en programas (Emiliani, 2004), en la creación de universidades Lean (Alp, 2001 y Hines \& Lethbridge, 2008) o en la mejora de procesos para reducir costos o incrementar eficiencias en procesos administrativos de universidades (Hines \&

2 Se entiende el término input como la información que "entra" o es requerida y que inicia todo el proceso de mejoramiento. 
Lethbridge, 2008), reduciendo los tiempos en el proceso de admisión de estudiantes o reprocesos en la información a estos, o procurando involucrar a toda la administración en maneras eficientes de desarrollo de procesos de instituciones de educación universitaria (Dew, 2007).

Principalmente en el campo de la educación es donde cobra importancia el hecho de hacer entender Lean como filosofía, como cultura (Atkinson, 2010). Es a través de las mismas personas que van a hacer uso del servicio o de quienes lo van a prestar, que se puede constituir un verdadero modelo de mejoramiento de los procesos de servicio.

Si se continúa asumiendo que Lean es solo una "caja de herramientas" o, peor aún, una simple herramienta de eliminación de costos, es imposible lograr algún tipo de cambio. También es necesario eliminar la concepción de que es algo que solo funciona en Japón "dada su cultura y el contexto allí existente" (Atkinson, 2010).

Lean Services, como filosofía de mejoramiento, requiere del análisis de toda la información disponible acerca de los procesos que se buscan mejorar, con el fin de analizarlos e identificar la manera como obtener los resultados esperados, tanto por el cliente externo como por la organización como tal.

Como parte de ese proceso de recolección de información, muchas de las empresas que han implementado Lean Thinking (ya sea para manufactura o servicios), se centran en la recolección interna de datos mediante el uso de diferentes metodologías para la identificación del valor agregado; de esta manera, se sesga el proceso completo de rediseño de los procesos a partir del concepto del cliente interno y se dejan de lado las percepciones y expectativas del cliente externo, quien finalmente es quien tomará la decisión de comprar el bien y/o servicio que la empresa vende. Sin embargo, esto no quiere decir que el uso de las herramientas de recolección de información de índole interna no aplique, sino que se deben complementar con los datos externos.

Al ser uno de los pilares de Lean la especificación del valor esperado por "todos" (clientes internos, externos y dueños o inversionistas), para luego crear ese valor esperado disminuyendo y/o reduciendo los desperdicios en el sistema, como lo explican Womack y Jones (2003), desde el concepto de CRM y la filosofía Lean es pertinente establecer que el eje central que define el valor agregado de los procesos es el cliente, quien reconoce cuáles son valiosos, ya que responden a sus necesidades y expectativas, y por los que está dispuesto a pagar más. 
La identificación de los procesos que aportan valor agregado al cliente se logra después de la realización del levantamiento y documentación de los procesos actuales, en conjunto con la aplicación adicional de técnicas investigativas que permiten recoger la percepción de los clientes. Es así como el mantenimiento y/o mejoramiento de los momentos de interacción que el cliente recuerda, representan los puntos sobre los que reconoce el valor agregado del proceso.

Asimismo, desde CRM la comunicación y aporte del cliente no se limita a la recepción de información sobre él mismo, aunque este ya es un componente que a la mayoría de las organizaciones les puede resultar innovador. CRM plantea la conformación de un diálogo permanente que permita a la organización modificar continuamente su oferta de acuerdo a las necesidades manifiestas de los usuarios, y es constante en ambos sentidos. Con este objetivo, a continuación se presenta la propuesta de la metodología que permitirá realizar un diagnóstico desde CRM en organizaciones prestadoras de servicios académicos, para una posterior implementación de Lean Services.

\subsection{Componentes de la metodología de diagnóstico desde CRM}

Luego del análisis de los casos documentados, se retoman los siguientes componentes para la implementación de la metodología de diagnóstico desde CRM en organizaciones prestadoras de servicios académicos (Voelkl, Silva, Solano\& Pulido, 2009), con el objeto de que los resultados arrojados por esta generen la información inicial necesaria que soporte los procesos de mejoramiento basado en la filosofía Lean. En la figura 2 se resumen los pasos de la metodología que se explican a continuación:

1. Declaración de compromiso por parte de la organización, como punto clave para la aplicación de la metodología

De acuerdo con Mendoza (2006), el éxito de la implementación de CRM es concebirla como una filosofía de negocios, no como estrategias tecnológicas puntuales y a corto plazo. Esto implica en muchas organizaciones cambiar de forma de pensar, pasar de una organización enfocada a la producción u ofrecimiento de servicios a una orientada al cliente, es decir, orientada a darle a este lo que requiere, sea gracias a un producto o un servicio determinado, pero escuchando y entendiendo a cada cliente, ya que cada persona tiene expectativas y necesidades únicas y dinámicas que cambian con el 
tiempo y el momento que esté viviendo. Por lo anterior, la relación con el mercado no es masiva sino que tiende a la personalización y a la creación de relaciones uno-uno a largo plazo. En este punto se requiere apoyo tecnológico y las tecnologías de la información (IT, por sus siglas en inglés) se constituyen en esa herramienta, al permitir la recepción, almacenamiento, ordenamiento y recuperación de información de los datos de los clientes por parte de múltiples usuarios en diferentes momentos y al mismo tiempo, lo cual refleja la importancia de esta herramienta de apoyo para el mantenimiento de las relaciones con los clientes de la organización, que es el objetivo final de la filosofía CRM.

Figura 2

Metodología de implementación de CRM

Compromiso de adopción de CRM y LEAN, por parte de la organización

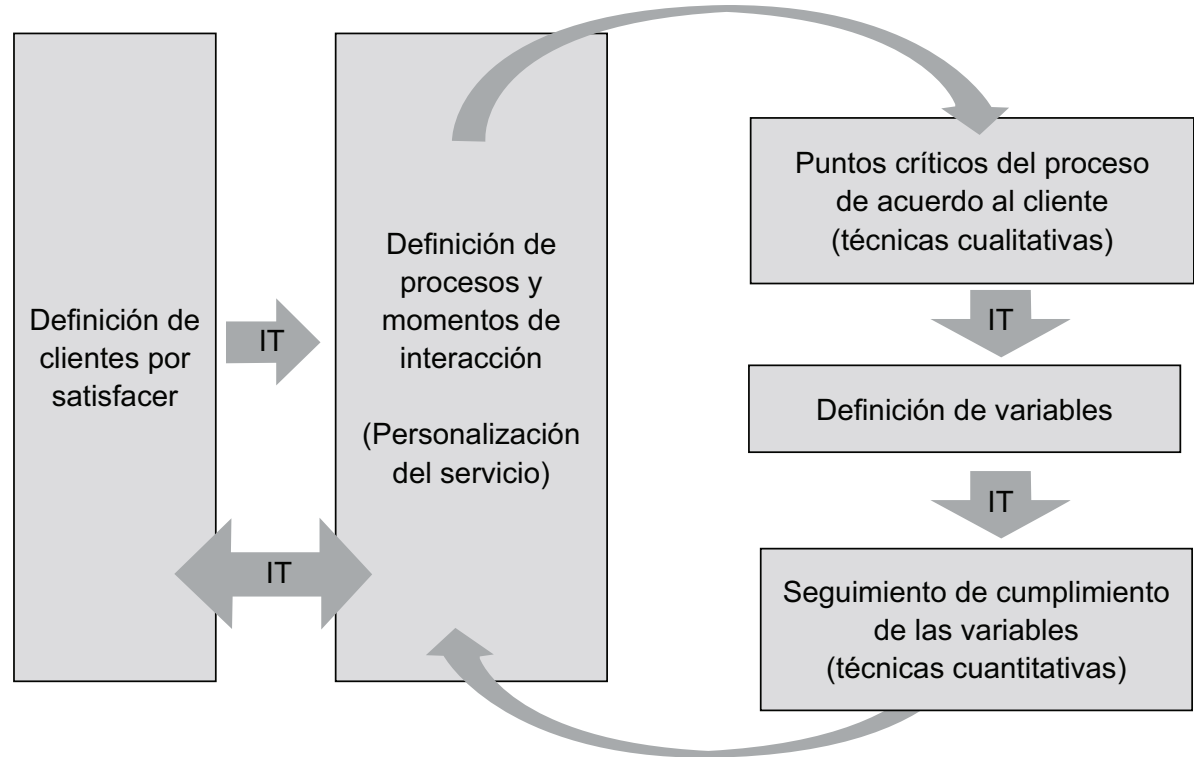

Fuente: Voelkl et al., 2009. 
Adicionalmente, el compromiso implica el apoyo del proceso desde los directivos hasta el empleado operativo, lo cual significa un proceso participativo en el que todas las personas de la organización estén involucradas con la finalidad de la empresa, que es satisfacer al cliente, enfocándose especialmente en los aspectos que —a juicio del cliente y no del proveedor-son más valiosos.

Como se mencionó, en el establecimiento del acuerdo de compromiso por parte de la organización, el cambio de orientación de la empresa implica pasar de la concepción de una empresa orientada a la producción a una orientada al cliente. En el ámbito de las instituciones académicas, esto implica, en muchos casos, reemplazar un paradigma educativo, en el cual la institución únicamente se enfoca a producir un servicio de educación, que a su juicio es de alta calidad académica, pero dejando de lado la producción de los otros servicios que van en forma paralela al proceso educativo y que son de índole administrativa, los cuales no suelen ser enfocados y orientados al cliente y a responder sus expectativas, sino en el cumplimiento de mínimos requerimientos organizacionales; asimismo, se olvida en algunos casos que la institución es una empresa, la cual debe ser sostenible y tener aspectos diferenciadores en el mercado que atraigan y mantengan a más clientes.

Este cambio ha sido trabajado en Europa ante las exigencias establecidas en el pacto de Barcelona en 1999 (E\&T, 2006) y no ha sido nada fácil, lo cual no se refleja de manera general en otros países, pues en la práctica aún se mantienen los esquemas educativos tradicionales.

Ante esta mirada, el estudiante se considera a sí mismo un actor pasivo, con el cual no se cuenta para mantener ningún tipo de relación a largo plazo ni como ente clave para retroalimentar el servicio educativo, por lo que el primer cambio que propone esta metodología de diagnóstico del sector educativo es el cambio de la mirada tradicional a la constructivista, donde el eje es el aprendizaje y el estudiante. En los aspectos administrativos propios de la organización, esta pasividad del cliente es claramente observable. Las instituciones diseñan procesos y los estudiantes se limitan a cumplirlos por obligación, ya que en muchos casos se perciben como "indispensables" para la organización, mas no para el estudiante. 
2. Identificación de los perfiles de los clientes a los cuales satisfacer Para establecer la relación uno a uno, se requiere definir a los clientes a los que la organización va a satisfacer. Este aspecto es el resultado de un análisis del negocio y del portafolio de servicios de la organización, de acuerdo a sus intereses actuales y futuros. Es decir, este aspecto está definido inicialmente con carácter institucional y formalmente en los documentos que soportan la planeación estratégica de la empresa (Serna, 2003). En este orden de ideas, las directivas de las instituciones definirán los clientes objetivos de acuerdo con los perfiles definidos, considerando la información que se tenga de estos actualmente. El ideal propuesto es considerar el ciclo de vida de los estudiantes, categorizarlos de acuerdo con este y definir sus características y expectativas.

3. Conocimiento de los procesos actuales y de los puntos de interacción con el cliente

A partir del diagnóstico inicial de la empresa, donde se identifican los procesos actuales de esta, y de la identificación inicial del cliente establecida en la planeación estratégica, se establece el perfil del cliente objetivo, el cual a través de investigaciones (mediante la aplicación de técnicas cualitativas y cuantitativas) se complementa y amplía continuamente, gracias a la información que constantemente se está recibiendo del cliente a través de los canales de comunicación establecidos por CRM.

Posteriormente, y sobre la base de los procesos actuales, se propone la identificación de los puntos de interacción con el cliente (o momentos de verdad), los puntos del proceso que él percibe como valiosos. Para lo anterior se utilizan herramientas propias de Lean como el Service Blueprinting, VSM (Value Stream Mapping) o el esquema de servicios, que permiten definir no solo los puntos de interacción con el cliente sino los procesos de apoyo.

4. Ampliación del perfil de los clientes gracias a la identificación de las expectativas manifestadas en los puntos de interacción

Este paso se realiza en paralelo con el tercero, ya que como resultado de identificar las percepciones y expectativas de los clientes en relación con los procesos, específicamente los momentos de interacción, y considerando que el cliente valora todo el proceso y a la empresa en sí por su experiencia en estos momentos, el cuarto punto es el resultado de indagar de manera más amplia y sistemática sobre 
lo anterior de forma constante y continua, apoyado en el sistema de información y en los canales establecidos desde CRM.

5. Revisión de la capacidad de respuesta de la organización frente a las expectativas de los clientes externos y definición de aspectos de mejoramiento en los procesos

El quinto paso busca equilibrar las expectativas del cliente y las posibilidades concretas de la empresa de responderlas. La filosofía CRM pretende lograr este equilibrio mediante acuerdos dialogados con cada uno de los clientes, donde las promesas de la empresa sean explícitas y concretas, pero flexibles y acordes con las expectativas del cliente. Inicialmente se sugiere que este paso se realice con los clientes actuales de mayor importancia para la organización y posteriormente se generalice. Este punto implica, por lo tanto, la personalización del servicio, de acuerdo con la capacidad de la organización frente a las expectativas de los clientes, a partir del diálogo continuo con estos, no desde el mero análisis del proveedor.

6. Definición de mecanismos de comunicación en doble vía con los clientes

Se propone que estos mecanismos se centren en los puntos de interacción, ya que se constituyen como la fuente de seguimiento y posteriormente como los indicadores de control de las propuestas de mejoramiento. Para la implementación de este punto, la definición de las tecnologías de información disponibles por la organización es vital, como soporte del proceso.

7. Aplicación Lean para el mejoramiento de los procesos de acuerdo a la identificación de los momentos de verdad, interacción o touch points que generan valor agregado

Una vez que se hayan identificado los puntos de mejora, respecto a la capacidad de respuesta que tiene la organización, se busca priorizar, de acuerdo con la percepción del cliente, los puntos en los que se es más crítica una respuesta acorde a sus expectativas, o donde la respuesta sea de vital importancia en la percepción adecuada del servicio por parte de este. Después de esta priorización se empezará a dar respuesta a la filosofía Lean, que como se ha mencionado se enfoca en las actividades que generan un valor agregado, en este caso de acuerdo con los requerimientos manifestados por el cliente gracias a la metodología de diagnóstico planteada; teniendo en cuenta la naturaleza de la situación encontrada y por mejorar, se deberá 
plantear un proceso de mejora a través del ciclo de implementación Lean, tal como lo explica Voelkl (2008), mediante las herramientas adecuadas para llegar, al menos, a los puntos concertados con el cliente.

Dentro del proceso de mejoramiento, deberán establecerse indicadores y acciones de seguimiento encauzadas a aumentar la calificación por parte del cliente, basados en la mejora del servicio y la capacidad de respuesta que se tenga como unidad administrativa. Se plantea que los indicadores tengan una meta alcanzable y que sean al mismo tiempo un reto para la unidad. Estos indicadores y metas deberán ser fijados por el equipo de trabajo sobre la base de las directrices de los líderes y las expectativas que a su vez manifiestan los clientes, priorizándolos.

Por otro lado, es pertinente que cada una de las áreas centre sus esfuerzos en los momentos de interacción y las características del servicio que los clientes consideran más importantes, es decir, las que para ellos generan valor.

El seguimiento de estos momentos críticos podrá ser evaluado a través de otros parámetros o métricas que en el futuro permitirán comparar el estado actual con el propuesto. Además, mediante el uso de herramientas de Lean, como el Value Stream Mapping (VSM) y el Service Blueprinting, se llegará a definir cómo y qué herramientas adicionales se tendrán que implementar para lograr los resultados esperados. Esto corresponde a la segunda etapa de esta investigación.

\subsection{Validación y aplicación de la metodología de diagnóstico propuesta}

Después de retomar el enfoque de CRM y de usar la metodología de diagnóstico desde esta filosofía en organizaciones prestadoras de servicios académicos (Voelkl et al., 2009) se procedió a validar la propuesta metodológica aplicándola como caso de estudio a los procesos administrativos de la carrera de Ingeniería Industrial de la Pontificia Universidad Javeriana ${ }^{3}$, evidenciando con su apoyo financiero la declaración

3 La metodología de implementación de CRM se aplicó en la carrera de Ingeniería Industrial de la Pontificia Universidad Javeriana dentro del proyecto de investigación "Diseño de una metodología de diagnóstico basado en CRM, como insumo de Lean Services para la definición del valor agregado requerido por los 
de compromiso de la institución por el interés de adoptar las filosofías Lean y CRM. Ello se constituye como el paso inicial y clave de la metodología. De igual forma, como resultado del segundo paso, se identificaron los perfiles de los cinco usuarios que se iban a satisfacer, que fueron los siguientes: estudiante activo vinculado al Programa de Práctica Social, empresa vinculada al Programa de Práctica Social, estudiantes activos vinculados al Programa de Práctica Profesional, empresas representadas por el jefe inmediato y/o interlocutor de Recursos Humanos vinculado al Programa de Práctica Profesional, y estudiantes activos.

Posteriormente, con la identificación y documentación de cada uno de los procedimientos administrativos de la carrera, así como el de las personas involucradas en cada uno de ellos se ubicaron, definieron y documentaron los procesos y procedimientos mediante la identificación del mapa de procesos y ejecución de una matriz de control de la documentación. Como resultado se encontraron 50 puntos de valor agregado en 9 procesos, los cuales se revisaron en el 2012 como resultado de la aplicación de validación cíclica y continua de la metodología.

De hecho, gracias a la aplicación de las técnicas cualitativas (sesiones de grupo y entrevistas en profundidad) y cuantitativas (encuestas) de forma sistemática, se estableció por parte del usuario el nivel de satisfacción relacionado con cada uno de los nueve procesos administrativos de la carrera.

Para lo anterior se realizaron 11 entrevistas y 5 sesiones de grupo para confirmar la existencia de los puntos de valor agregados identificados en la primera aplicación de la metodología y posteriormente se aplicaron 757 encuestas para determinar el grado de conformidad con los puntos de valor agregado con un nivel de confianza del $95 \%$. El instrumento solicitó a los usuarios la medición de la conformidad mediante una escala de cinco puntos, donde 1 es "total desacuerdo" y 5 "total acuerdo".

usuarios de la carrera de Ingeniería Industrial de la PUJ, Bogotá" realizada por los autores en el 2007 y apoyada por la Vicerrectoría Académica con el número 002935, y en el 2012, en el proyecto "Lean aplicado a procesos administrativos en Instituciones de Educación Superior. Caso: carrera de Ingeniería Industrial de la Pontificia Universidad Javeriana", identificado en SIAP con el código 004904. 
Posteriormente, se agruparon los resultados en tres categorías, donde los valores 1 y 2 se asignaron a la categoría "malo", los valores 3 y 4 a la categoría "bueno" y en la categoría "excelente" se asignó la valoración de 5 en la escala, lo cual permitió a la institución identificar la percepción de los usuarios. Con esta información, considerando el número de usuarios en cada proceso, así como el número de puntos de valor agregados por ellos, se estableció que los procesos cuyo mejoramiento a través de Lean generarían un mayor impacto positivo en la valoración de los usuarios son los relacionados con Práctica Social y Práctica Profesional.

Se procedió, entonces, a jerarquizar los procedimientos cuya pronta intervención tendrían impacto inmediato y sobre los cuales se deben focalizar los esfuerzos y recursos. Para esto, se consideró el valor acumulado de las categorías "mala" y "buena" identificando así los puntos de valor agregado que se deben mejorar mediante la aplicación de la filosofía y conceptos Lean como aquellos que presentaban $60 \%$ o más de inconformidad. Los aspectos identificados se muestran en la tabla 1.

Tabla 1

Aspectos identificados priorizados

\begin{tabular}{l} 
Área de Práctica Social \\
\hline - Realización del taller de Habilidades Sociales \\
- Realización del taller de Fundamentación \\
- Humanística \\
- Realización de los seminarios de facultad, foro social \\
- Reausura del programa \\
- Realización de la reunión informativa \\
- Realización de la inducción general \\
- Asignación de proyectos a estudiantes \\
- Realización de seguimiento permanente \\
- Realización de reunión académica \\
- Recepción, registro y entrega de informes \\
- Actualización de formatos y encuestas de evaluación \\
\hline
\end{tabular}

(continúa) 


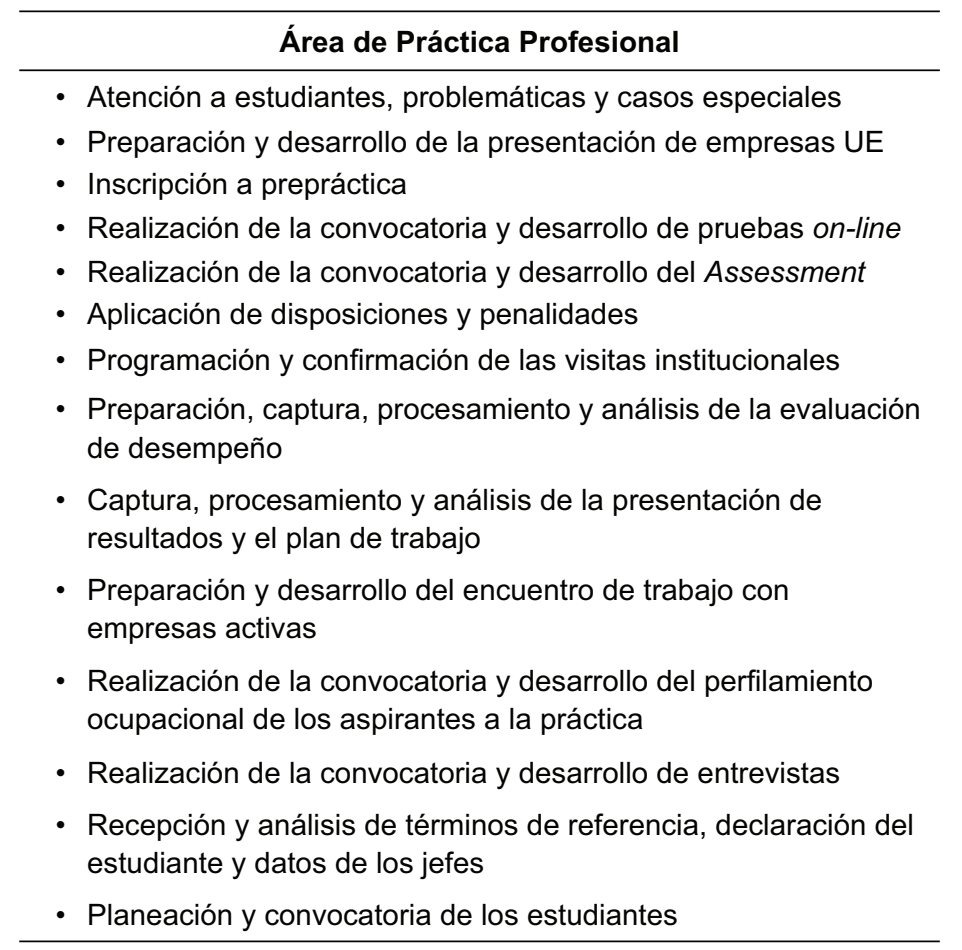

Elaboración propia.

Una vez traducidas las percepciones de los clientes externos en términos de los procedimientos correspondientes, se procede a aplicar las diferentes herramientas de revisión interna de la filosofía Lean, en especial mediante el uso de la herramienta Value Stream Mapping (VSM), con la que se identifican tanto los desperdicios y oportunidades de mejora, así como se analizan algunas métricas e indicadores cuantitativos, como el diagnóstico de tiempos y cargas de trabajo, a lo largo de cada uno de los procedimientos. Algunas de estas métricas son las comúnmente utilizadas para procesos de servicio, como lo mencionan Keyte y Locher (2004) y Balzer (2010).

Por otro lado, en forma paralela, se usan matrices de análisis de desperdicios mudas por actividad, según el modelo utilizado por Balzer (2010), mediante la clasificación general de desperdicios de Savendy y Karkowski (2010); de esta forma, se identifican los desperdicios desde el punto de vista cuantitativo y desde el análisis cualitativo, donde se 
incluyen los valiosos comentarios de los clientes externos que hacen parte de los sentimientos y percepciones que difícilmente pueden ser medidos y trabajados desde el VSM.

A partir de esta información y análisis global del diagnóstico, se pueden definir las herramientas y conceptos de Lean Thinking que podrán implementarse para el mejoramiento de los procesos y que lograrán adicionalmente lo esperado por los clientes externos, como fin principal de Lean.

\section{CONCLUSIONES}

Retomando el hecho de que Lean Services es una filosofía inscrita dentro de la filosofía principal de Lean Thinking, que busca la disminución y/o eliminación de los desperdicios, no solo a través del flujo de material sino de información, especialmente, en el caso de Lean Services, el enfoque de esta se dirige al mejoramiento del flujo de información, con la inclusión de los medios físicos y tecnológicos para que se lleve a cabo el intercambio de información de la manera más efectiva. Precisamente, para realizar actividades que generen valor agregado y procesos orientados a satisfacer las necesidades de los clientes, Lean Services utiliza diferentes herramientas de diagnóstico en forma interna tanto de la empresa como de los propios procesos; sin embargo, estas no involucran directamente al cliente externo de las organizaciones. Adicionalmente, para el proceso de diseño y/o rediseño de los procesos, aunque Lean Services propugna como etapa inicial el análisis de los requerimientos de los clientes, esta filosofía no cuenta con una herramienta metodológicamente diseñada para identificar el valor agregado percibido por los clientes externos a partir de sus propias expectativas y requerimientos, sino que utiliza investigaciones de mercado puntales, que no se mantienen actualizadas.

Por otro lado, el uso práctico del concepto de CRM ha sido útil para identificar y dar respuesta oportuna a las expectativas de los clientes de manera continua, entre otros beneficios demostrados en las exitosas aplicaciones de diversos sectores empresariales. De la misma forma, aunque CRM ha sido aplicado en algunas instituciones de educación superior, este no ha sido utilizado para analizar el valor agregado que una institución o unidad académica esté generando, desde la percepción del cliente externo, para este caso, estudiantes potenciales y activos, y empresas asociadas a las prácticas. 
Como filosofía de negocio, CRM es más que la aplicación de tecnologías de información, lo que implica utilizar no solo el apoyo informático, sino también la información para personalizar la oferta al cliente, centrándose en la identificación de lo que es percibido por este como valor agregado del proceso y por lo que finalmente paga. Para lograr su adecuado "uso" se requiere que la organización reconozca su misión, visión, objetivos y capacidades, ya que de acuerdo con este conocimiento identifica a los clientes que son de su interés y justifican, como negocio, estar en comunicación constante para la personalización de sus servicios.

Los beneficios para la organización deben constituir igualmente un beneficio para los clientes, que a largo plazo impliquen su fidelización. Esto se logra manteniendo los canales de comunicación en doble vía y, por ende, la personalización de los servicios. La relación a largo plazo, mencionada anteriormente, reconoce el ciclo de vida del consumidor y pretende "servirlo y escucharlo" en cada momento. Esta relación se transforma en fidelización, al representar para el cliente una mayor inversión el cambio de proveedor, al tener que iniciar con este una relación desde cero.

Para las entidades de educación superior o instituciones académicas adoptar el CRM como una filosofía de negocios implica un doble esfuerzo, puesto que desde diferentes enfoques educativos, la educación no se percibe abiertamente como una relación de negocios donde se presentan los proveedores, los clientes y el servicio como tal. De acuerdo con Hilbert, Schonbrunn y Schmode (2007), este enfoque ha cambiado en países europeos y en Estados Unidos especialmente, dada la situación de competencia en que estas operan. Esta nueva concepción ha sido positiva precisamente porque ha implicado orientarse hacia los estudiantes potenciales, actuales, egresados y empresas vinculadas, las cuales en determinado momento del ciclo de vida del cliente son la razón de ser de las universidades específicamente.

Es claro, entonces, que la implementación de Lean para que sea orientada al cliente requiere contacto con este y un mecanismo para establecer una comunicación permanente, continua, ágil y en doble vía, característica del CRM.

Dado lo anterior, se propone la siguiente metodología de diagnóstico desde CRM como input Lean, sintetizándola en los siguientes componentes: 
1. Declaración de compromiso por parte de la organización como punto clave para la aplicación de la metodología.

2. Identificación de los perfiles de los clientes que se van a satisfacer.

3. Conocimiento de los procesos actuales y de los puntos de interacción con el cliente.

4. Ampliación del perfil de los clientes gracias a la identificación de las expectativas manifestadas en los puntos de interacción.

5. Revisión de la capacidad de respuesta de la organización frente a las expectativas de los clientes externos y definición de aspectos de mejoramiento en los procesos.

6. Definición de mecanismos de comunicación en doble vía con los clientes.

7. Aplicación Lean para el mejoramiento de los procesos de acuerdo con la identificación de los momentos de verdad, interacción o touch points que generan valor agregado.

A partir de este diagnóstico del valor esperado por los clientes a través de los momentos de interacción identificados, lo que se busca desde la filosofía Lean es centrarse en satisfacer las expectativas de los clientes, en las actividades percibidas como generadoras de valor agregado al servicio ofrecido, dirigiendo los esfuerzos hacia el cumplimiento de los cinco fundamentos de esta filosofía:

1. Especificar el valor esperado.

2. Identificar el flujo de valor.

3. Crear un flujo de valor.

4. Halar a través del sistema.

5. Luchar por la perfección.

Es importante mencionar que con la implementación de la propuesta metodológica planteada en este proyecto y la validación realizada en el caso de estudio, se cumpliría con los numerales 1,2 y parte del 3 , lo cual es valioso, ya que incluye la perspectiva de los clientes externos y no solo una mirada subjetiva de la experiencia y percepción de quien realiza el proceso, operación o actividad. Esta especificación es el punto de partida del que se continuaría con la metodología para la implementación de la filosofía Lean en cualquier institución académica.

De esta forma, gracias al diálogo o contacto permanente en ambos sentidos con el cliente, se utiliza el CRM como filosofía base para la 
metodología de diagnóstico propuesta, al identificar estos puntos críticos y valorados por el cliente para que sean mejorados con la aplicación de Lean.

Por otro lado, como se puede observar en los componentes propuestos, por pertenecer a la implementación de la filosofía, se presentan como pasos que se recomienda aplicar constante y cíclicamente, para actualizar permanentemente la información de los clientes y la adecuación de los procesos internos de la organización, siempre ante las exigencias continuas y cambiantes del medioambiente. Esta revisión constante se sugiere que se realice en periodos y frecuencias programadas. En la práctica implica, gracias al apoyo de las tecnologías de información, la definición y configuración de un sistema que soporte este manejo de información, el cual podría basarse en la plataforma con que la institución académica cuente en el momento de su implementación.

Es importante recordar que cada organización, como ente independiente y único, responde a comportamientos y estilos gerenciales propios, dada su visión y misión estratégica. Este aspecto es reconocido igualmente por los clientes como un valor agregado que genera confianza, aspecto clave desde CRM. De igual forma, cabe recalcar lo esencial: no solo se debe "escuchar" al cliente, almacenar estos datos, organizarlos y recuperarlos, CRM va más allá, pues implica un compromiso de la organización hacia los clientes, por dar una respuesta a esta información reflejada en la personalización del servicio; es ahí donde la aplicación de Lean permite responder al cliente en forma productiva, al optimizar la ejecución de los procesos.

Dentro de la propuesta de mejoramiento desde la filosofía Lean se deben priorizar los esfuerzos para lograr mejoras concretas y puntuales que puedan ser percibidas en un corto-mediano plazo por los clientes, con el propósito de que encuentren una respuesta a sus expectativas y comentarios dentro del proceso de mejoramiento. Estas propuestas tendrán que ir dirigidas hacia los procesos y momentos de interacción identificados como críticos para los clientes.

\section{REFERENCIAS}

Abdi, F., Shavarini, S. K., \& Hoseini, S. G. (2006). Lean: How to use Lean approach in Service industries. Journal of Services Research, 6 [edición especial]. 
Alp, N. (2001). The Lean transformational model for the education system. International Conference of Computers and Industrial Engineering Conference Proceedings. Montreal: The University of Tennessee at Chattanooga.

Atkinson, P. (2010). Lean is a cultural isuue. Management Services Magazine, 54, issue 2, 35-51.

Balzer, W. K. (2010). Lean higher education: increading the value and performance of university processes. Nueva York: Productivity Press.

Beasty, C. (2005). A college learns a thing or two from CRM in Customer Relationship Management. ABI/Inform Global. 9(6), 52.

Broady-Preston, J., Felice, J., \& Marshal, S. (2006). Building better customer relationships: case studies drom Malta and the UK. Emerald Library Management, 27(6/7), 430-445.

Dew, J. (2007). Quality goes to college. Quality Progress, 45(48), 50-52.

Education \& Training (E \& T). (2006). La enseñanza superior en Europa. Recuperado de http://ec.europa.eu/education/policies/ educ/higher/higher_es.html

Emiliani, M. L. (2004). Improving business school courses by applying Lean principles and practices. Quality Assurance in Education 12(4).

Hilbert, A., Schonbrunn, K., \& Schmode, S. (2007). Student relationship management in Germany: foundations and opportunities. Management Review - ABI/Inform Global, 18(2), 204.

Hines, P., \& Lethbridge, S. (2008). New development: creating a Lean university. Public Money and Management, 28, issue 1, 53-56.

Keyte, B., \& Locher, D. (2004). The complete Lean enterprise: value stream mapping for administrative and office processes. New York: Productivity Press.

Liker, J. K., \& Morgan, J. M. (2006). The Toyota way in services: the case of lean product development. Academy of Management Perspectives Journal, 20, 5-20.

Lin, Y., Su Hwan-Yann, \& Chien, S. (2005). A knowledge-enabled procedure for customer relationship management. Industrial Marketing Management, 35, 446-456. 
Mendoza, L., Marius, A., Pérez, M., \& Grimán, A. (2006). Critical Success factors for a customer relationship management strategy. Science Direct, 49, 913-945.

Pan, S., Tan, C., \& Lim, E. (2004). Customer relationship management in e-government: a relational perspective. Decision support systems, 42, 237-250.

Roshayati, A. H. (2011). Factors influencing the success of Lean services implementation conceptual framework. Second International Confrence on Buisiness and Economic Research (ICBER). Recuperado de http://www.internationalconference. com.my/proceeding/2ndicber2011_proceeding/272-2nd\%20 ICBER\%202011\%20PG\%201496-1509\%20Lean\%20Services\%20 Implementation.pdf

Salyendy, G., \& Karkowski, W. (2010). Introduction to service engineering. Noboken-New Jersey: John Wiley \& Sons Inc.

Serna, E. (2003). Gerencia estratégica teoría-metodología, alineamiento, implementación y mapas estratégicos, índices de gestión. Bogotá: $3 \mathrm{R}$ editores.

Voelkl, J. R. (agosto, 2008). Application of lean principles in academic support, focused in the current programme of industrial engineering (school of engineering) at the Pontificia Universidad Javeriana, Bogotá-Colombia. Ponencia presentada en The 3rd World Conference of Production and Operations Management (POMS). Organizado por POMS (Production and Operations Managemet Society) and JOMSA (Japanese Operations Management and Strategy Association).

Voelkl, J. R., Silva, J. A., Solano, C. M., \& Pulido, L. M. (2009). Enfoque CRM, percepciones de los usuarios de los procesos administrativos académicos y su valor agregado. Revista Universidad EAFIT, 34(156), 90-103.

Womack, J. P., \& Jones, D. T. (2003). Lean thinking vs Muda. LondonGreat Britain: Simon \& Schuster. 